\title{
A COUNTEREXAMPLE IN THE THEORY OF FINITE GROUPS ${ }^{1}$
}

\author{
DAVID A. SIBLEY
}

\begin{abstract}
Suppose $G$ is a finite group and $p$ and $q$ are distinct odd primes. Let $\chi$ be an irreducible character of $G$ and $x$ and $y$ be a $p$-element and a $q$-element of $G$ such that $\chi(x), \chi(y)$ are both irrational. In this situation it is known that $G$ contains an element of order $p q$. John Thompson has asked whether $y$ must commute with some conjugate of $x$. We show by example that this need not be the case.
\end{abstract}

H. F. Blichfeldt [1, Theorem 13] discovered the following remarkable theorem.

Theorem. Suppose $G$ is a finite group and $p$ and $q$ are distinct odd prime divisors of $|G|$. Let $x$ and $y$ be a p-element and a q-element of $G$. If $G$ has an irreducible complex character $\chi$ for which both $\chi(x)$ and $\chi(y)$ are irrational, then $G$ contains an element of order $p q$.

This result was generalized by W. Burnside [2, Chapter XVI, Theorem X]. The reader is referred to [3, Theorem (6.13)] for the statement of Burnside's theorem and a proof due to R. Brauer. According to [3, p. 41], J. Thompson has asked whether, in the situation of the theorem, $y$ must commute with some conjugate of $x$. If true, this would produce a natural element of order $p q$. However, we shall answer Thompson's question in the negative. The author is indebted to J. Alperin of the University of Chicago for invaluable advice in this construction.

We first construct the group $G$. Let $p$ be any prime such that $p-1$ has a prime divisor $q \geqslant 5$. Let $P$ denote the upper triangular Sylow $p$-group of GL $(4, p)$. Thus, elements of $P$ have diagonal entries 1 , zeros below the diagonal, and arbitrary entries from $\mathrm{GF}(p)$ above the diagonal. Clearly $|P|=p^{6}$. Since $q \mid p-1$ and $q \geqslant 5$, we can find $a, b \in \mathrm{GF}(p)$ such that $a, b$, and $a b^{-1}$ are distinct primitive $q$ th roots of 1 . Let $y$ be the diagonal matrix $\operatorname{diag}(1, a, b, 1)$, so $|y|=q$, and let $G$ be the subgroup of $\operatorname{GL}(4, p)$ generated by $y$ and $P$. Then $|G|=p^{6} q$ and $P \triangle G$. A straightforward calculation shows that the center $Z$ of $P$ has order $p$ and satisfies $C_{P}(y)=Z$. Thus, $Z$ is also the center of $G$.

Received by the editors February 16, 1976.

AMS (MOS) subject classifications (1970). Primary 20C15; Secondary $20 \mathrm{H} 20$.

${ }^{1}$ Research supported by NSF Grant MPS 75-06334. 
Lemma 1. Suppose $\varphi$ is a faithful irreducible complex character of $P$. Then there is an $x \in P-Z$ with $\varphi(x)$ irrational.

Proof. We first show that there is an $x_{0} \in P-Z$ with $\varphi\left(x_{0}\right) \neq 0$. Indeed, if there is no such $x_{0}$, we write

$$
\begin{aligned}
1 & =\|\varphi\|_{P}^{2}=\frac{1}{p^{6}} \sum_{g \in P}|\varphi(g)|^{2} \\
& =\frac{1}{p^{6}} \sum_{g \in Z} \varphi(1)^{2}=\frac{1}{p^{5}} \varphi(1)^{2} .
\end{aligned}
$$

Since $\varphi(1)$ is an integer, $\varphi(1)^{2}=p^{5}$ is absurd. Thus, $x_{0}$ exists as claimed. Now, if $\varphi\left(x_{0}\right)$ is irrational, choose $x=x_{0}$. If $\varphi\left(x_{0}\right)$ is rational, choose $z \in Z^{\#}$ and set $x=x_{0} z$. Since $\varphi(x)=\varphi\left(x_{0}\right) \varphi(z) / \varphi(1)$ and $\varphi(z)$ is irrational, we have $\varphi(x)$ irrational in this case as well.

LEMMA 2. $G$ has a faithful irreducible complex character $\chi$ satisfying both

(1) $\chi \mid P$ is faithful and irreducible,

(2) $\chi(y)$ is irrational.

Proof. We first apply Brauer's matrix duality theorem [3, Theorem (12.1)] to $y$ acting on the character table of $P$. Since $y$ centralizes $Z$, this theorem shows there is an irreducible character $\varphi$ of $P$ which is $G$-invariant and has $Z \mathbb{E} \operatorname{ker} \varphi$. Since $Z$ is the center of $P$ and $|Z|=p$, we have $\operatorname{ker} \varphi=1$. That is, $\varphi$ is faithful. Since $\varphi$ is $G$-invariant and $|G: P|=q$ is prime, there is an irreducible character $\chi_{0}$ of $G$ with $\chi_{0} \mid P=\varphi$. Note that $\chi_{0}(y) \neq 0$, for otherwise $0=\chi_{0}(y) \equiv \chi_{0}(1)(\bmod q)$ and $\chi_{0}(1)=\varphi(1)$ is a power of $p$, a contradiction. If $\chi_{0}(y)$ is irrational, choose $\chi=\chi_{0}$. If $\chi_{0}(y)$ is rational, let $\lambda$ be a nonprincipal linear character of $G / P$ and set $\chi=\lambda \chi_{0}$.

It is now clear that $G$ is the desired counterexample. By Lemma $2(2), \chi(y)$ is irrational and by Lemma 2(1) and Lemma $1, \chi(x)$ is irrational for some $x \in P-Z$. However, $C_{P}(y)=Z$ and $x \in P-Z$, so $y$ does not commute with any conjugate of $x$.

\section{REFERENCES}

1. H. F. Blichfeldt, On the order of linear homogeneous groups. II, Trans. Amer. Math. Soc. 5 (1904), 310-325.

2. W. Burnside, Theory of groups of finite order, 2nd ed., Dover, New York, 1955 (reprint). MR 16, 1086.

3. W. Feit, Characters of finite groups, Benjamin, New York, 1967. MR 36 \#2715.

Department of Mathematics, Pennsylvania State University, University Park, PennSYLVANIA 16802 